\title{
Lexicographic semigroupoids
}

\author{
S. C. POWER \\ Department of Mathematics and Statistics, Lancaster University, Lancaster LAI 4YF, \\ England
}

(Received 7 March 1994)

\begin{abstract}
The natural lexicographic semigroupoids associated with Cantor product spaces indexed by countable linear orders are classified. Applications are given to the classification of triangular operator algebras which are direct limits of upper-triangular matrix algebras.
\end{abstract}

\section{Introduction}

Consider a Cantor space which is presented explicitly as an infinite product of finite topological spaces. The product presentation provides an equivalence relation $R$ consisting of the pairs $(x, y)$ of points $x$ and $y$ which disagree in at most finitely many coordinates. This equivalence relation supports a natural locally compact totally disconnected topology which makes $R$ a principal groupoid. It is well-known that in the case of countable products such topological equivalence relations are classified by the generalised integer obtained from the formal product of the cardinalities of the component spaces. Furthermore, this classification is closely related to the classification of $\mathrm{C}^{*}$-algebras that are infinite tensor products of matrix algebras, the so-called UHF $\mathrm{C}^{*}$-algebras. See, for example, Renault [10] and Power [7].

In the present paper we consider antisymmetric topological binary relations which are the lexicographic topological subrelations arising from infinite products indexed by general countable linear orderings. These natural semigroupoids are classified and their automorphism groups determined. This and related results enable us to give applications to the classification of triangular operator algebras which are themselves lexicographic products in an algebraic sense.

The binary relations may also be viewed as the (semigroupoid) lexicographic products of total orderings on finite sets, and in fact our methods are applicable to lexicographic products of connected antisymmetric finite partial orders. Although applications to approximately finite operator algebras provide our primary motivation, it seems clear that lexicographic subrelations are interesting in their own right.

In $\S 1$ we recall how the generalised integer associated with the presentation of the Cantor space gives a complete invariant for the associated approximately finite groupoid. In $\S 2$ we classify the lexicographic products in the case of indexing by a countable dense 
order. It is interesting that the proof here is much more elementary than the case of indexing by $\mathbb{Z}$ which is taken up in $\S 3$. Indeed, the classification in the former case is less subtle and suggests, a posteriori, that the component coordinates must be accessible in purely order-topological terms. The proof in $\S 3$ follows the order-topological methods in Power [8] where automorphisms of the associated triangular algebras were studied. These algebras-the so-called alternation algebras-have been considered by a number of authors, namely Poon [6], Hopenwasser and Power [4], and most recently, as part of a wider analysis, by Donsig and Hopenwasser [3]. Also in $\S 3$ we identify the automorphism group of a lexicographic product over $\mathbb{Z}$. In $\S 4$ we obtain the classification for the case of general countable linear orders and in the last section we give applications to operator algebras.

\section{Preliminaries}

Let $\mu$ be a positive integer-valued function defined on a countable linear ordering $\Omega$. For notational convenience we denote the discrete finite topological space $\{1, \ldots, n\}$ by $[n]$. Associate with $\mu$ the Cantor space

$$
X_{\mu}=\prod_{w \in \Omega}\left[r_{w}\right]
$$

where $r_{w}=\mu(w)$. Write $\tilde{R}_{\mu}$ for the equivalence relation described $\S 0$. Let $R_{\mu}$ be the antisymmetric subrelation of points $(x, y) \in \tilde{R}_{\mu}$, for which $x$ preceeds $y$ in the lexicographic ordering. Thus $(y, x) \in R_{\mu}$ if and only if $x=\left(x_{w}\right)$ and $y=\left(y_{w}\right)$, the coordinates $\left(x_{w}\right)$ and $\left(y_{w}\right)$ are equal except in at most a finite number of coordinates, and $y_{w}<x_{w}$ for the first index $w$ where $x$ and $y$ differ.

The basic open-closed sets for the Cantor space $X_{\mu}$ are provided by the cylinder sets arising from the specification of a finite number of coordinates. The topology on $\tilde{R}_{\mu}$ arises in the following similar way. Let $F \subseteq \Omega$ be a finite subset, let $x_{w}^{\prime}$, $y_{w}^{\prime}$ be specified coordinates for $w \in F$, and let

$$
E=\left\{(x, y): x_{w}=y_{w} \text { for } w \notin F, x_{w}=x_{w}^{\prime} \text { and } y_{w}=y_{w}^{\prime} \text { for } w \in F\right\}
$$

The totality of these sets gives a base for the groupoid topology on $\tilde{R}_{\mu}$ and the subrelation $R_{\mu}$ carries the relative topology.

Notice that if $\pi_{l}, \pi_{r}: \tilde{R}_{\mu} \rightarrow X_{\mu}$ are the natural coordinate projections then $\pi_{l}$ and $\pi_{r}$ are continuous and are one-to-one when restricted to a basic open-closed set $E$. General open-closed sets with this property are called $G$-sets and these are important in the following two ways. Firstly they provide a class of sets which are invariant under groupoid isomorphism, that is, a binary relation isomorphism that is also a homeomorphism. Secondly they are used in the formulation of invariant measures on the underlying space. As a consequence groupoid isomorphisms conserve the invariant measures.

An invariant measure for a principal groupoid $\tilde{R}$ is a Borel measure $\lambda$ for the underlying topological space such that $\lambda\left(\pi_{l}(G)\right)=\lambda\left(\pi_{r}(G)\right)$ for every $G$-set $G$. It is a simple matter to check that $\tilde{R}_{\mu}$ possesses a unique invariant probability measure, namely the infinite product of the probability measures $\lambda_{\omega}$ on $\left[r_{\omega}\right]$ which assign equal mass $r_{\omega}^{-1}$ to 
each singleton set. At this stage we can deduce that if $\tilde{R}_{\mu}$ is isomorphic to the topological equivalence relation $\tilde{R}_{v}$, associated with $\nu: \Lambda \rightarrow \mathbb{N}$, then the generalised integers for $\mu$ and $\nu$ agree. Indeed, the hypothesised isomorphism is a bijection $\alpha: X_{\mu} \rightarrow X_{\nu}$ such that the map $\alpha^{(2)}: \tilde{R}_{\mu} \rightarrow \tilde{R}_{\nu}$ is a bijection and a topological isomorphism. Since the invariant probability measures for $\tilde{R}_{\mu}$ and $\tilde{R}_{\nu}$ are unique they must correspond under $\alpha$, and from this it follows that they have the same range on open-closed sets. Thus the set of rationals $k / r$, with $k \in \mathbb{Z}$ and $r=r_{w_{1}} r_{w_{2}} \cdots r_{w_{n}}$ for some $n \in \mathbb{N}$, coincides with the corresponding set of rationals for $v$. Equivalently

$$
\prod_{w \in \Omega} \mu(w)=\prod_{w \in \Lambda} v(w)
$$

as generalised integers.

In the antisymmetric context we shall use the argument given above in a local way (with various restrictions of $R_{\mu}$ in place of $\tilde{R}_{\mu}$ ) to obtain local comparisons of the data for two given lexicographic binary relations.

For $x$ in $X_{\mu}$ the (one-sided) orbit of $x$ is the set $\mathcal{O}(x)$ of points $y$ with $(y, x) \in R_{\mu}$, and the closed orbit of $x$ is $\overline{\mathcal{O}(x)}$ the closure of this set. Note that if $x=\left(x_{w}\right)$ with $x_{w}=1$ for all $w \leq w_{1}$ and $x_{w}=\mu(w)$ for all $w>w_{1}$, for some $w_{1}$ in $\Omega$, then $\overline{\mathcal{O}(x)}$ is the set of points $y=\left(y_{w}\right)$ with $y_{w}=1$ for $w \leq w_{1}$. This will be indicated by setting $y_{w_{1}}=1$ and writing

$$
\overline{\mathcal{O}(x)}=\left(. .1 . .\left|y_{w_{1}}\right| . . * . .\right)
$$

A similar such shorthand is used in the next section to indicate basic open-closed sets in $X_{\mu}$. Note that for the particular point $x$ above the restriction $R_{\mu} \mid \overline{\mathcal{O}(x)}$ is isomorphic to a lexicographic ordering.

\section{Countable dense orders}

In this section we classify the topological binary relations $R_{\mu}$ in the cases when $\Omega$ is a dense linear ordering and $\mu: \Omega \rightarrow \mathbb{N}$ satisfies $\mu(w) \geq 2$ for all $w$ in $\Omega$. There are only four such linear orderings and these correspond to the presence and absence of maximal and minimal elements.

THEOREM 1. Let $\mu: \Omega \rightarrow \mathbb{N} \backslash\{1\}$ and $\nu: \Omega^{\prime} \rightarrow \mathbb{N} \backslash\{1\}$ be functions on the countable dense linear orderings $\Omega$ and $\Omega^{\prime}$. Then the lexicographic semigroupoids $R_{\mu}$ and $R_{\nu}$ are isomorphic if and only if there is an order bijection $\pi: \Omega \rightarrow \Omega^{\prime}$ such that $\nu(\pi(w))=\mu(w)$ for all $w$ in $\Omega$.

Proof. Assume first that $\Omega$ and $\Omega^{\prime}$ have no minimal elements.

Observe that a point $x=\left(x_{w}\right)$ in $X_{\mu}$ has a closed orbit $\overline{\mathcal{O}(x)}$ which is a proper subset of $X_{\mu}$ if and only if there exists an index of $t$ in $\Omega$ such that $x_{w}=1$ for all $w \leq t$. Indeed, assume that this does not hold and consider an arbitrary point $y$ in $X_{\mu}$. For an index $s \in \Omega$, with $x_{s}>1$, let $z^{s}=\left(z_{w}\right)$ where $z_{w}=y_{w}$ for $w<s, z_{s}=x_{s}-1$, and $z_{w}=x_{w}$ for $w>s$. If $A$ is a basic open neighbourhood of $y$ then, from the assumption, it follows that there exists an element $z^{s}$ in $A$. Since $\left(z^{s}, x\right) \in R_{\mu}$ the observation follows. 
Divide the set of points with proper closed orbit into two types. A point $X$ is of type 1 if there exists a first index $w_{1}$ such that $x_{w_{1}} \neq 1$, and is of type 2 otherwise. We claim that $x$ is type 1 if and only if $\overline{\mathcal{O}(x)}$ contains a closed orbit $\overline{\mathcal{O}(y)}$ which is a relatively open-closed proper subset. This property identifies the indexing in order topological terms and is the basis of the proof.

If $x$ is a type 2 point then there is an order interval decompositions $\Omega=\Omega_{1}+\Omega_{2}$, where $\Omega_{2}$ has no first element, such that $y$ belongs to the closed orbit of $x$ if and only if $y=\left(y_{w}\right)$ with $y_{w}=1$ for $w \in \Omega_{1}$. To see this let $\Omega_{1}$ be the largest order ideal in $\Omega$ of indices $w$ with $x_{w}=1$. Thus $\Omega=\Omega_{1}+\Omega_{2}$, where $\Omega_{2}$ has no first element and for any index $u$ in $\Omega_{2}$ there is a smaller index $v$ with $x_{v}>1$. In particular $\overline{\mathcal{O}(x)}$ contains the set

$$
\left(. .1 . .\left|x_{v}-1\right| . . * . .\right) \text {. }
$$

The union of all such sets is dense in the set

$$
\overline{\mathcal{O}(x)}=(. .1 . . \mid . . * . .)
$$

associated with the decomposition of $\Omega$. It follows that $\overline{\mathcal{O}(x)}$ is precisely this latter set, as required.

A relatively open-closed subset of $\overline{\mathcal{O}(x)}$ contains a basic relatively open-closed neighbourhood of the form

$$
\left(. .1 . .|. * . .| z_{w_{1}}|. . * . .| z_{w_{2}}|. . * . ., \ldots, . . * . .| z_{w_{n}} \mid . . * . .\right)
$$

with $w_{1}<w_{2}<\cdots<w_{n}$. Such a set contains points $z=\left(z_{w}\right)$ with $z_{t}=2$ for all $t$ in $\Omega_{2}$ with $t<w_{1}$. Since $\overline{\mathcal{O}(z)}=\overline{\mathcal{O}(x)}$ for such a point it follows that $\overline{\mathcal{O}(x)}$ cannot contain, properly, a relatively open-closed closed orbit.

We now identify the integers $\mu(w)$ in order-topological terms.

Let $E \subseteq X_{\mu}$ be an intersection of closed orbits of type 2 points which is not itself a closed orbit of a type 2 point. Then, in view of the description above of the closed orbits of type 2 points, $E$ has the form

$$
E^{(q)}=\bigcup_{i=1}^{\mu(q)}(. .1 . .|i| . . * \ldots),
$$

for some $q$ in $\Omega$. From this set and the relation $R_{\mu}$ we can discover $\mu(q)$ in ordertopological terms as follows. There is a unique $R_{\mu}$-invariant probability measure, $\lambda$ say, on the set $E^{(q)}$. The sets

$$
E_{k}^{(q)}=\bigcup_{i=1}^{k}(. .1 . .|i| . . * . .)
$$

are the only closed orbits contained in $E^{(q)}$ with positive $\lambda$ measure, and the reciprocal of the measure of the smallest such set is $\mu(q)$.

Suppose now that $\alpha^{(2)}: R_{\mu} \rightarrow R_{\nu}$ is a semigroupoid isomorphism implemented by $\alpha: X_{\mu} \rightarrow X_{\nu}$. In particular $\alpha$ is a homeomorphism. If $p \in \Omega^{\prime}$ then write $E^{(p)}$ for the sets in $X_{\nu}$ that are analogous to the subsets $E^{(q)}$. Then from the paragraph above it follows that $\alpha\left(E^{(q)}\right)=E^{\pi(q)}$ for some element $\pi(q)$ in $\Omega^{\prime}$. The map $\pi$ is an order isomorphism and from the characterisation of the numbers $\mu(q)$ above it follows that $\nu(\pi(q))=\mu(q)$ for all $q$ in $\Omega$. 
Suppose, finally, that $\Omega$ has a minimal element $w_{0}$. Then all points of $X_{\mu}$ except the unique maximal point $x_{\max }=(\mu(w))$ have proper closed orbits. Also, the converse is true. Note that there exist open-closed orbits and a smallest open-closed orbit, namely

$$
(1 \mid . * \ldots) \text {. }
$$

Since this set is determined in order-topological terms we can restrict considerations to this subset of $X_{\mu}$ and deduce the theorem in this case from the one already considered.

\section{The case $\Omega=\omega^{*}+\omega$}

A classification is now given for the lexicographic semigroupoids $R_{\mu}$ in the case where, in the standard notation, $\Omega$ has order type $\omega^{*}+\omega$. This order (integer) type is quite a bit more subtle than that of the dense orders in that semigroupoid isomorphisms may have to be effected by homeomorphisms defined in terms of a recoding.

Throughout this section assume that $\Omega=\mathbb{Z} \backslash\{0\}$ and that $\mu, v$ are maps from $\Omega$ to $\mathbb{Z}_{+}$with

$$
\mu(k)=r_{k}, \quad \mu(-k)=s_{k}, \quad \nu(k)=t_{k}, \quad \nu(-k)=u_{k},
$$

for $k=1,2, \ldots$ Associate with $\mu$ the pair $(\underline{r}, \underline{s})$ of generalised integers

$$
\underline{r}=r_{1} r_{2} \cdots, \quad \underline{s}=s_{1} s_{2} \cdots .
$$

Define an equivalence relation $\sim$ on pairs of generalised (or finite) integers by $(r, \underline{s}) \sim$ $(\underline{t}, \underline{u})$ if and only if $\underline{r s}=\underline{t u}$ and there exist coprime natural numbers $a, b$ such that $b \underline{r}=a \underline{t}$ and $a \underline{s}=b \underline{u}$.

THEOREM 2. Let $\mu, v: \Omega \rightarrow \mathbb{N}$, with associated pairs of generalised integers (possibly finite) $(\underline{r}, \underline{s})$ and $(\underline{t}, \underline{u})$ respectively. Then the lexicographic semigroupoids $R_{\mu}$ and $R_{v}$ are isomorphic if and only if $(\underline{r}, \underline{s}) \sim(t, \underline{u})$.

Proof. We prove the necessity of the condition for isomorphism. The sufficiency direction is relatively straightforward and is left to the reader. (See also [4] and [6].)

Let $\alpha: X_{\mu} \rightarrow X_{\nu}$ be a bijection such that $\alpha^{(2)}: R_{\mu} \rightarrow R_{\nu}$ is a topological isomorphism. The inversion map $\theta: X_{\mu} \times X_{\mu} \rightarrow X_{\mu} \times X_{\mu}$ given by $\theta\left(\left(x, x^{\prime}\right)\right)=\left(x^{\prime}, x\right)$ is an automorphism of the equivalence relation $\tilde{R}_{\mu}$, and $\tilde{R}_{\mu}=R_{\mu} \cup \theta\left(R_{\mu}\right)$. It follows that $\alpha^{(2)}$ maps $\tilde{R}_{\mu}$ homeomorphically onto $\tilde{R}_{\nu}$. In particular, by the discussion in $\S 1$, it follows that the generalised integer for $\tilde{R}_{\mu}$ coincides with that for $\tilde{R}_{\nu}$. That is, $\underline{r} \underline{s}=\underline{t} \underline{u}$.

Let $X_{\mu, 0} \subseteq X_{\mu}$ be the set of points $x=\left(x_{k}\right)$ with $x_{k}=1$ for sufficiently small $k$. If $X_{\mu, 0}$ is a proper subset of $X_{\mu}$ then it is precisely the set of points in $X_{\mu}$ with proper closed orbits, and so $\alpha\left(X_{\mu, 0}\right)=X_{\nu, 0}$. The set $X_{\mu, 0}$ contains the special points for which, in addition, $x_{k}=r_{k}$ for all sufficiently large $k$. These special points can be characterised order topologically. Indeed they are precisely the points $x$ for which there exists a point $x^{+}$whose closed orbit $\overline{\mathcal{O}\left(x^{+}\right)}$is the union of $\{x+\}$ and $\overline{\mathcal{O}(x)}$. These so-called gap points are discussed in [4] and [7]. Thus, if $x_{*}=\left(\ldots, 1,1, \hat{r}_{1}, r_{2}, \ldots\right)$, where the symbol indicates the coordinate position $k=1$, then $\alpha\left(x_{*}\right)$ may be written as

$$
\left(\ldots, 1,1, w_{-j+1}, w_{-j}, \ldots, w_{j-1}, u_{j}, u_{j+1}, \ldots\right)
$$


for some positive integer $j$. We have

$$
\overline{\mathcal{O}\left(\boldsymbol{x}_{*}\right)}=(\ldots, 1,1, \hat{*}, *, \ldots) \text {, }
$$

and

$$
\overline{\mathcal{O}\left(\alpha\left(x_{*}\right)\right)}=\left\{\left(\ldots, 1, w^{\prime}, y_{j}, y_{j+1}, \ldots\right): w^{\prime} \in\left[1_{j}, w\right]\right\}
$$

where $y_{k} \leq u_{k}$ for $k \geq j$, and where $w^{\prime}$ is any word of length $2 j-2$ which preceeds, or is equal to, the word $w=\left(w_{-j+1}, \ldots, w_{j-1}\right)$. That is, $w^{\prime}$ belongs to the lexicographic order interval $\left[1_{j}, w\right]$ where $1_{j}$ is the word with $2 j-2$ coordinates all equal to 1 . Let $n$ be the number of words in this order interval and note that the restricted topological equivalence relation $\tilde{R}_{v} \mid \overline{\mathcal{O}\left(\alpha\left(x_{*}\right)\right)}$ has generalised integer $n t_{j} t_{j+1} \cdots$. Since $\alpha$ induces an isomorphism between this relation and the topological equivalence relation $\tilde{R}_{\mu} \mid \overline{\mathcal{O}\left(x_{*}\right)}$ then, once again, by the discussion in $\S 1$, we have $n t_{j} t_{j+1} \cdots=r_{1} r_{2} \cdots$, and hence $m \underline{r}=n \underline{t}$ where $m=t_{1} t_{2} \cdots t_{j-1}$. Although $\underline{r s}=\underline{t u}$ we cannot yet conclude that $(\underline{r}, \underline{s}) \sim(\underline{t}, \underline{u})$.

Define $d_{\mu}: X_{\mu, 0} \rightarrow \mathbb{R}$ by

$$
d_{\mu}\left(x_{k}\right)=\sum_{k=1}^{\infty} \frac{x_{k}-1}{r_{1} r_{2} \cdots r_{k}}+\sum_{k=1}^{\infty}\left(x_{-k}-1\right) s_{0} s_{1} \cdots s_{k-1},
$$

where $s_{0}=1$, and define $d_{\nu}: X_{\nu, 0} \rightarrow \mathbb{R}$ similarly. If $x \in \overline{\mathcal{O}\left(x_{*}\right)}$ then we can interpret $d_{\mu}(x)$ as the measure of $\overline{\mathcal{O}(x)}$ with respect to the unique normalised $R_{\mu}$-invariant Borel measure on $\overline{\mathcal{O}\left(x_{*}\right)}$. Call this measure $\lambda_{\mu}$, and note that there is a unique $\tilde{R}_{\mu}$-invariant extension to $X_{\mu, 0}$ which we also denote by $\lambda_{\mu}$.

Because of the uniqueness of normalised invariant measures it follows that $\lambda_{\nu} \circ \alpha=$ $c \lambda_{\mu}$ and $d_{\nu}(\alpha(x))=c d_{\mu}(x)$ for some positive constant $c$. Since $d_{\mu}\left(x_{*}\right)=1$ we have $c=d_{\nu}\left(\alpha\left(x_{*}\right)\right)$. To see that $c=n / m$ note that there are precisely $m$ points in the product $\left[t_{1}\right] \times \cdots \times\left[t_{j-1}\right]$. Thus, from the definition of $n$ and the product measure $\lambda_{\nu}$ we see that $\lambda_{\nu}\left(\overline{\mathcal{O}\left(\alpha\left(x_{*}\right)\right)}\right)=n / m$.

We now use the connection $d_{\nu}(\alpha(x))=c d_{\nu}(x)$ to show that $n \underline{s}=m \underline{u}$. We can assume that $\underline{s}$ and $\underline{u}$ are not finite.

Let $n / m=a / b$ where $(a, b)=1$ and suppose, by way of contradiction, that $a \underline{s}$ does not divide $b \underline{u}$. Then, there exists a prime number $p$ and a positive integer $g$ such that

$$
p^{g}\left|a \underline{s}, \quad p^{g-1}\right| b \underline{u}, \quad \text { and } \quad p^{g} \not b \underline{u} .
$$

Choose $l$ large enough so that $a s_{1} \cdots s_{l}=k_{1} p^{g}$ for some integer $k_{1}$. Then

$$
k_{1} \frac{b}{a} \frac{u_{1} u_{2} \cdots u_{l}}{s_{1} s_{2} \cdots s_{l}}=b \frac{u_{1}^{\prime} u_{2}^{\prime} \cdots u_{l}^{\prime}}{p}
$$

and

$$
\left(p, b u_{1}^{\prime} u_{2}^{\prime} \cdots u_{l}^{\prime} u_{l+1} \cdots u_{v}\right)=1
$$

for all $v>l$, where $u_{1}^{\prime}, \ldots, u_{l}^{\prime}$ are factors of $u_{1}, \ldots, u_{l}$ respectively. Note that by increasing $l$, if necessary, and compensating with a multiple of $k_{1}$, we can arrange that the product $s=s_{1} s_{2} \cdots s_{l}$ satisfies $p^{-1}<1-s^{-1}$. These numerical relations lead to the contradiction that $\alpha(E)=X_{\nu}$ where $E$ is the proper open-closed subset

$$
E=\bigcup_{w \in W}(\ldots * \ldots, w, \ldots * \ldots)
$$


where $W$ is the set of words $\left(s_{l}^{\prime}, \ldots, s_{1}^{\prime}\right)$ which are strictly less than $\left(s_{l}, s_{l-1}, \ldots, s_{1}\right)$ in the lexicographic ordering.

To see this let $y$ be an arbitrary point of $X_{v}$ and let $F_{v}(y)$ be the closed set

$$
F_{v}(y)=\left\{y^{\prime} \in X_{\nu}: y^{\prime}=\left(y_{k}^{\prime}\right), y_{k}^{\prime}=y_{k} \text { for } k \geq-v\right\}
$$

for $v=1,2, \ldots$ The range of $d_{\nu}$ on $F_{\nu}(y) \cap X_{\nu, 0}$ is an arithmetic progression, namely,

$$
d_{\nu}\left(F_{v}(y) \cap X_{\nu, 0}\right)=\left\{k u_{1} u_{2} \cdots u_{v}+\xi: k \in \mathbb{Z}_{+}\right\}
$$

where $\xi=d_{v}\left(y^{+}\right)$, and where $y^{+}$agrees with $y$ in coordinates indexed by $\mathbb{Z}_{+}$and is equal to $l$ in the remaining coordinates.

On the other hand we have

$$
d_{\mu}\left(E \cap X_{\nu, 0}\right)=\bigcup_{k=1}^{\infty}[k s, k s+(s-1)]
$$

and so

$$
d_{\nu}\left(\alpha(E) \cap X_{\mu, 0}\right)=\bigcup_{k=1}^{\infty}[c k s, c k s+c(s-1)] .
$$

In particular, since $c=a / b$, the set $d_{\nu}\left(F_{\nu}(y) \cap X_{\nu, 0}\right)$ meets $d_{\nu}\left(\alpha(E) \cap X_{\nu, 0}\right)$ if and only if the sets $B_{v}$ and $A$ meet, where

$$
A=\bigcup_{k=1}^{\infty}\left[k, k+\left(1-s^{-1}\right)\right]
$$

and

$$
B_{v}=\left\{k \frac{b}{a} \frac{u_{1} u_{2} \cdots u_{v}}{s}+\frac{\xi}{s}: k \in \mathbb{Z}_{+}\right\} .
$$

By our earlier remarks, and our choice of $l$, if $v>l$ then $B_{v}$ contains the set

$$
B_{v}^{\prime}=\left\{k \frac{b u_{1}^{\prime} u_{2}^{\prime} \cdots u_{l}^{\prime} u_{l+1} \cdots u_{v}}{p}+\frac{\xi}{s}: k \in \mathbb{Z}_{+}\right\} .
$$

By the coprimality of $p$ and $b u_{1}^{\prime} u_{2}^{\prime} \cdots u_{v}$ this set contains certain positive integral translates of the points

$$
\frac{u}{p}+\frac{\xi}{s}, \quad \text { for } i=1, \ldots, p .
$$

Since $p^{-1}<\left(1-s^{-1}\right)$ it follows that $B_{v}^{\prime}$ meets the set $A$. We have thus shown that

$$
d_{v}\left(F_{v}(y) \cap X_{\nu, 0}\right) \cap d_{v}\left(\alpha(E) \cap X_{\nu, 0}\right) \neq \emptyset
$$

and hence, by the openness of $\alpha(E)$, that

$$
F_{v}(y) \cap \alpha(E) \neq \emptyset, \quad \text { for } v=1,2, \ldots
$$

Since $y$ is the unique point in the intersection of the sets $F_{v}(y)$, it follows that $y \in \alpha(E)$ and hence that $\alpha(E)=X_{v}$, the desired contradiction.

We have shown that $a \underline{s}$ divides $b \underline{u}$. Since $\alpha^{-1}$ is also an automorphism we conclude that $b \underline{u}$ divides $a \underline{s}$ and hence that $b \underline{u}=a \underline{s}$, and $(\underline{r}, \underline{s}) \sim(t, \underline{u})$. 
Remark 1. Note that from the proof above it follows that the restrictions $R_{\mu} \mid X_{\mu, 0}$ and $R_{\nu} \mid X_{\nu, 0}$ are isomorphic topological binary relatations if and only if $R_{\mu}$ and $R_{\nu}$ are isomorphic topological binary relations. We use this fact in the next section.

Remark 2. As we mention later, there is a close association between approximately finite topological binary relations and approximately finite triangular operator algebras. The algebras associated with the $\mathbb{Z}$-ordered relations above correspond to the socalled alternation algebras considered by Hopenwasser and Power [4] and by Poon [6]. Their classification is also given, as part of a more general study, by Donsig and Hopenwasser [3]. The argument we give above follows very closely the method of [8], which was restricted to the case $\mu=v$. (There is an inadequacy in the arithmetic progression argument of [8] which is corrected in the somewhat more general argument above.)

Remark 3. In principle it should be possible to reformulate the arguments of Poon [6] and Donsig and Hopenwasser [3] in terms of binary relations to give alternative proofs of Theorem 2. The arguments we have given are also suited to other situations and in particular to the generalised alternation algebras associated with Markov chains and subshifts. (See [7].) We intend to report more fully on this elsewhere. However, let us note the following example from [7].

Let $R_{\mu}$ be the lexicographic topological binary relation for $\Omega=\mathbb{Z}$ with the function $\mu(w)=2$ for all $w$. Let $R_{1}$ (resp. $R_{2}$ ) be the topological subrelation defined on the symbol subspace $X_{1}$ (resp. $X_{2}$ ) of $X_{\mu}$ for which the pair 00 (resp. 11) is forbidden. Then, in contrast to their generated equivalence relations, $R_{1}$ and $R_{2}$ are not isomorphic. Similarly the one-sided subrelations are not isomorphic.

Automorphisms. Fix $\mu: \Omega \rightarrow \mathbb{N}$ as above with lexicographic semigroupoid $R_{\mu}$ and generalised integer pair $(\underline{r}, \underline{s})$. Let $d$ be the number of primes $p$ such that both $\underline{r}$ and $\underline{s}$ are divisible by $p^{\infty}$.

THEOREM 3. [8] The semigroupoid automorphism group Aut $\left(R_{\mu}\right)$ is isomorphic to the restricted direct product $\mathbb{Z}^{d}$.

Proof. An element $\alpha^{(2)} \in \operatorname{Aut}\left(R_{\mu}\right)$ is a topological isomorphism induced by a bijection $\alpha: X_{\mu} \rightarrow X_{\mu}$. By the argument in the proof of Theorem 2, specialized to the case $\mu=\nu$, we have

$$
d_{\mu}(\alpha(x))=c d_{\mu}(x)
$$

for $x \in X_{\mu, 0}$, where $c=a / b$ with $(a, b)=1$, $a \underline{r}=b \underline{r}$, and $b \underline{s}=a \underline{s}$. Furthermore, if $c=1$ then $\alpha$ is trivial. These conditions imply that $a^{\infty}$ and $b^{\infty}$ divide $\underline{r}$ and $\underline{s}$. It follows that $c$ has the form $p_{1}^{e_{1}} p_{2}^{e_{2}} \cdots p_{k}^{e_{k}}$ where $e_{i} \in \mathbb{Z}$ and each $p_{i}$ divides $\underline{r}$ and $\underline{s}$ with infinite multiplicity. The mapping $\alpha \rightarrow c$ gives the desired isomorphism.

Suppose that $p$ is a prime such that $p^{\infty}$ divides $\underline{r}$ and $\underline{s}$. We note one way in which the order-preserving homeomorphism $\alpha$ corresponding to $p^{-1}$ may be identified. 
Write $\underline{r}=p r_{1} p r_{2} p r_{3} \cdots$ and $\underline{s}=s_{1} p s_{2} p s_{3} \cdots$ and obtain the identification

$$
\begin{array}{ccc}
X_{\mu} & = & \left(\cdots\left[s_{2}\right] \times[p] \times\left[s_{1}\right]\right) \times\left([p] \times\left[r_{1}\right] \times[p] \times \cdots\right) \\
& = & X_{\mu}^{-} \times X_{\mu}^{+} .
\end{array}
$$

This is naturally isomorphic to

$$
\begin{array}{rlc}
X_{\lambda} & = & \left(\cdots[p] \times\left[s_{1}\right] \times[p]\right) \times\left(\left[r_{1}\right] \times[p] \times\left[r_{2}\right] \times \cdots\right) \\
& = & X_{\lambda}^{1} \times X_{\lambda}^{+}
\end{array}
$$

by a map $\beta=\beta^{-} \times \beta^{+}$which respects the factors and which induces the natural semigroupoid isomorphism $R_{\mu} \rightarrow R_{\mu}$. Let $\gamma: X_{\lambda} \rightarrow X_{\mu}$ be the right shift homeomorphism. Then $\gamma \circ \beta$ is an automorphism of $X_{\mu}$ and its associated constant is $p^{-1}$.

\section{Countable linear orderings}

Let $\Omega$ be a countable linear ordering and define an equivalence relation $\approx$ on $\Omega$ such that $w \approx v$ if the order intervals $[w, v]$ and $[v, w]$ are finite. Then the set $\Omega / \approx$ of equivalence classes is linearly ordered and each equivalence class $\langle x\rangle$ is itself a linearly ordered set which is isomorphic to a finite set or to one of $\mathbb{Z}_{+}, \mathbb{Z}_{-}$and $\mathbb{Z}$. Let $\mu: \Omega \rightarrow\{2,3, \ldots\}$. Then to each class $\langle x\rangle$ we can associate a pair $p_{\mu}(\langle x\rangle)=(\underline{r}, \underline{s})$, as in $\S 3$, consisting of finite or generalized integers.

THEOREM 4. Let $\Omega, \Lambda$ be countable linear orderings with maps $\mu: \Omega \rightarrow\{2,3, \ldots\}$, $v: \Lambda \rightarrow\{2,3, \ldots\}$. Then the lexicographic semigroupoids $R_{\mu}$ and $R_{\nu}$ are isomorphic if and only if there is an order preserving bijection $\pi: \Omega / \approx \rightarrow \Lambda / \approx$ such that $p_{\mu}(\pi(\langle w\rangle)) \sim p_{\mu}(\langle w\rangle)$ for all classes $\langle w\rangle$ in $\Omega / \approx$.

Proof. As in the proof of Theorem 1, declare a point $x=\left(x_{w}\right)$ in $X_{\mu}$ to be a type 1 point if there exists a first coordinate $w$ for which $x_{w} \neq 1$. Once again, as in the proof of Theorem 1, these points are identifiable in order-topological terms as those for which the closed (half) orbit $\overline{\mathcal{O}(x)}$ properly contains relatively open-closed closed orbits. If $w_{2}>w_{1}$ then say that $w_{1}$ and $w_{2}$ are finitely equivalent if there exist type 1 points $x, y$ with first non-unit coordinates $x_{w_{1}}$ and $y_{w_{2}}$ respectively such that $\overline{\mathcal{O}(y)}$ has positive measure with respect to the unique invariant probability Borel measure on $\overline{\mathcal{O}(x)}$.

Note that $w_{1}$ and $w_{2}$ are finitely equivalent if and only if $w_{1} \approx w_{2}$. In view of this it follows that if $\alpha^{(2)}: R_{\mu} \rightarrow R_{\nu}$ is a topological isomorphism induced by the homeomorphism $\alpha$ then $\alpha$ induces a map $\pi: \Omega / \approx \rightarrow \Lambda / \approx$. Indeed, if $x$ is a type 1 point associated with $w$ in $\Omega$ then $\alpha(x)$ is a type 1 point associated with $u$ in $\Lambda$ and we may define $\pi(\langle w\rangle)=\langle u\rangle$. Since the equivalence relation $\approx$ coincides with finite equivalence this is a well-defined bijection.

Fix a class $\langle w\rangle$, for some $w \in \Omega$, and define the set

$$
X_{\mu}(\langle w\rangle)=\bigcup_{x \in T(w)} \overline{\mathcal{O}(x)}
$$

where the union is taken over the set $T(w)$ of type 1 points $x$ associated with the class $\langle w\rangle$. Then $\alpha$ restricts to a homeomorphism

$$
\beta: X_{\mu}(\langle w\rangle) \rightarrow X_{\nu}(\pi(\langle w\rangle))
$$


By considering the restriction of $\alpha^{(2)}$ to the set

$$
R_{\mu} \cap\left(X_{\mu}(\langle w\rangle) \times X_{\mu}(\langle w\rangle)\right)
$$

we shall show that the lexicographic semigroupoid $R(\langle w\rangle, \mu)$ associated with the linear order $\langle w\rangle$ and the function $\mu$ is isomorphic to $R(\pi(\langle w\rangle), v)$. In view of the result in $\S 3$ this will complete the proof.

To this end define the equivalence relation $E_{\mu}$ on $X_{\mu}(\langle w\rangle)$ as the set of pairs $(x, y)$ for which $x_{u}=y_{u}$ for all $u$ in $\langle w\rangle$, and similarly define $E_{v}$ on $X_{v}(\pi(\langle w\rangle))$. Thus, the set of equivalence classes $X_{\mu}(\langle w\rangle) / E_{\mu}$ is isomorphic to the set

$$
X_{\langle w\rangle}=\prod_{d \in\langle w\rangle}[\mu(d)] .
$$

Furthermore, $R_{\mu}$ and $E_{\mu}$ induce a binary relation, $R_{\mu} / E_{\mu}$ say, on the set $X_{\mu}(\langle w\rangle) / E_{\mu}$. That is $(x, y) \in R_{\mu} / E_{\mu}$ if and only if there exist $x^{\prime}, y^{\prime}$ in $X_{\mu}(\langle w\rangle)$ with $\left(x^{\prime}, y^{\prime}\right) \in R_{\mu}, x^{\prime} \in$ $x$ and $y^{\prime} \in y$. Also, under the natural identification above $R_{\mu} / E_{\mu}$ is the lexicographic semigroupoid $R(\langle w\rangle, \mu)$ on $X_{\langle w\rangle}$.

In view of these identifications it will be enough to show that the equivalence relation $E_{\mu}$ can be defined in an order-topological fashion and that the restriction of $\alpha$ to $X_{\mu}(\langle w\rangle)$ induces a semigroupoid isomorphism from $R(\langle w\rangle, \mu)$ to $R(\pi(\langle w\rangle), \mu)$.

In $\S 2$ we saw that, up to a constant multiplier, the set $X_{\mu}(\langle w\rangle)$ carries a unique $R_{\mu}$-invariant measure, $\lambda_{\mu}$ say. If $x, y \in X_{\mu}(\langle w\rangle)$ and $\lambda_{\mu}(\overline{\mathcal{O}(x)})=\lambda_{\mu}(\overline{\mathcal{O}(y)})$ then there are two possibilities; either $x_{u}=y_{u}$ for all $u$ in $\langle w\rangle$, or $x_{u}$ and $y_{u}$ correspond to 'rational points' in the sense that the symmetric difference $\overline{\mathcal{O}(x)} \Delta \overline{\mathcal{O}(y)}$ is a singleton, namely $\{x\}$ or $\{y\}$. It follows that $E_{\mu}$ can be defined purely in order-topological terms and hence that $\alpha$ maps the $E_{\mu}$-equivalence classes to $E_{\nu}$-equivalence classes.

Since the given map $\alpha: X_{\mu} \rightarrow X_{\nu}$ is continuous and since the basic open-closed sets of $X_{\mu}$ and $X_{\mu}$ generate the topology of $X_{\mu}$ and $X_{\mu}$, respectively, it follows that the induced map

$$
\alpha_{i}: X_{\mu}(\langle w\rangle) / E_{\mu} \rightarrow X_{\nu}(\pi(\langle w\rangle)) / E_{\nu}
$$

is bicontinuous. Indeed $\alpha$ maps an $E_{\mu}$-saturated basic open-closed set to an $E_{\nu}$-saturated open-closed set and this is necessarily a finite union of $E_{\nu}$-saturated basic open-closed sets. Similarly, it follows that $\alpha_{i}^{(2)}$ induces a semigroupoid isomorphism from $R(\langle w\rangle, \mu)$ to $R(\pi(\langle w\rangle), \mu)$.

The arguments given above and in the previous section are also effective in the setting of infinite lexicographic products of partially ordered sets. This is illustrated in the following theorem in the case $\Omega=\mathbb{Q}$ for which the proof in $\S 2$ is applicable with little change.

For each rational $q \in \mathbb{Q}$ let $\leq_{q}$ be a connected partial ordering on the finite set $\{1, \ldots, \mu(q)\}$. Then the product space $X_{\mu}$ carries a natural semigroupoid

$$
R=R\left(\left\{\leq_{q}: q \in \mathbb{Q}\right\}\right),
$$

which is the subset of $R_{\mu}$ associated with the given partial orderings. That is, $(x, y) \in R$ if and only if $(x, y) \in \tilde{R}_{\mu}$ and $x_{w_{0}} \leq_{q} y_{w_{0}}$ for the smallest index $w_{0}$ such that $x_{w} \neq y_{w}$. 
THEOREM 5. The semigroupoids $R\left(\left\{\leq_{q}: q \in \mathbb{Q}\right\}\right)$ and $R\left(\left\{\prec_{q}: q \in \mathbb{Q}\right\}\right)$ are isomorphic if and only if there is an order bijection $\pi: \mathbb{Q} \rightarrow \mathbb{Q}$ such that the partial orderings $\leq_{q}$ and $\prec_{\pi(q)}$ are isomorphic for all $q \in \mathbb{Q}$.

\section{Applications to operator algebras}

The operator algebra $T_{n}$ is the subalgebra of the complex matrix algebra $M_{n}$ consisting of upper-triangular complex matrices and endowed with the usual operator norm. These algebras $A$ are triangular in the sense that $A \cap A^{*}$ is a maximal abelian self-adjoint subalgebra. Recently there has been considerable interest in classifying the many diverse families of triangular operator algebras arising as direct limits of these finite-dimensional algebras and their direct sums (see [7]). These algebras can also be viewed as triangular subalgebras of groupoid $C^{*}$-algebras (see Renault [10] or Muhly and Solel [5]).

The following construction is given in [9].

Let $\Omega, \mu$ be as above, with $n_{w}=\mu(w)$. Let $F \subseteq \Omega$ be a finite subset, say $w_{1}<w_{2}<\cdots<w_{k}$, and let $w_{t}<w<w_{t+1}$, for some $t$. Set $G=F \cup\{w\}$, $n_{F}=n_{w_{1}} n_{w_{2}} \cdots n_{w_{k}}$, and $n_{G}=n_{\omega} n_{F}$. Define a unital algebra injection $\phi_{F, G}: T_{n_{F}} \rightarrow T_{n_{G}}$ as follows. View $T_{n_{F}}$ as the (maximal triangular) subalgebra of $M_{n_{w_{1}}} \otimes \cdots \otimes M_{n_{w_{k}}}$ which is spanned by the matrix units

$$
e_{\mathrm{i}, \mathrm{j}}=e_{i_{1}, j_{1}} \otimes \cdots \otimes e_{i_{k}, j_{k}},
$$

where the multi-index $\mathbf{i}=\left(i_{1}, \ldots, i_{k}\right)$ precedes $\mathbf{j}=\left(j_{1}, \ldots, j_{k}\right)$ in the lexicographic ordering. Thus either $\mathbf{i}=\mathbf{j}$ or the first $i_{p}$ differing from $j_{p}$ is strictly less than $j_{p}$. Similarly identify $T_{n_{G}}$ for the ordered subset $G$ and set $\phi_{F, G}$ to be the linear extension of the correspondence

$$
e_{\mathrm{i}, \mathrm{j}} \rightarrow \sum_{s=1}^{n_{\omega}} e_{i_{1}, j_{1}} \otimes \cdots \otimes e_{i_{i}, j_{t}} \otimes e_{s, s} \otimes e_{i_{t+1}, j_{l+1}} \otimes \cdots \otimes e_{i_{k}, j_{k}} .
$$

In a similar way, or by composing maps of the above type, define $\phi_{F, G}$ for $F \subseteq G$, general finite subsets. These maps are isometric and so determine the Banach algebra

$$
A(\Omega, v)=\lim _{\rightarrow F \in \mathcal{F}} T_{n_{F}},
$$

where the direct limit is taken over the directed set $\mathcal{F}$ of finite subsets of $\Omega$. Each $\phi_{F, G}$ has an extension to a $\mathrm{C}^{*}$-algebra injection from $M_{n_{F}}$ to $M_{n_{G}}$ and so it follows that we may view $A(\Omega, \nu)$ as a closed unital subalgebra of the UHF C*-algebra $B(\Omega, \nu)=\underset{\rightarrow}{\lim } M_{n_{F}}$.

THEOREM 6. The following statements are equivalent.

(i) $A(\Omega, \mu)$ and $A(\Lambda, \nu)$ are isometrically isomorphic Banach algebras.

(ii) $R_{\mu}$ and $R_{v}$ are isomorphic lexicographic semigroupoids.

(iii) There is an order preserving bijection $\pi: \Omega / \approx \rightarrow \Lambda / \approx$ such that

$$
p_{\mu}(\pi(\langle w\rangle)) \sim p_{\mu}(\langle w\rangle)
$$

for all classes $\langle w\rangle$ in $\Omega / \approx$. 
Proof. The semigroupoids are readily identifiable with the topological fundamental relations of the operator algebras. (See, for example, the discussions of [4] and [7].) The equivalence of (i) and (ii) is now immediate from Theorem 7.5 of [7] and so Theorem 4 completes the equivalences.

An immediate corollary of the last theorem is that there are uncountably many triangular algebras $A$ of the form $A(\Omega, \mu)$ with $C^{*}(A)$ equal to the $2^{\infty}$ UHF $\mathrm{C}^{*}$ algebra. On the other hand there are only three such (infinite-dimensional) algebras of the form $A(\mathbb{Z}, \mu)$, namely the pure refinement algebra, the standard limit algebra and the alternation algebra with invariant $\left(2^{\infty}, 2^{\infty}\right)$. We remark that if $\Omega$ fails to have a first element then the operator algebras $A(\Omega, \mu)$ have trivial Jacobson radical (see [2] and [9]).

We now explain how the algebras above can also be interpreted in term of a lexicographic product operation at the algebraic level, as described in [9].

Let $A$ be an operator algebra admitting a subdiagonal decomposition in the sense that

$$
A=A \cap A^{*}+A^{0}
$$

where $A \cap A^{*}$ is a maximal abelian subalgebra of $A$, and $A^{0}$ is the kernel of a contractive homomorphism $A \rightarrow A \cap A^{*}$. In particular, this holds if $A$ is a regular triangular subalgebra of an $A F C^{*}$-algebra ([7] or, more generally, if $A$ is a subdiagonal algebra in the sense of Arveson [1]). If $A$ and $B$ are triangular operator algebras admitting such decompositions then define their lexicographic product $A \star B$ to be the closed subalgebra of the injective tensor product $C^{*}(A) \otimes C^{*}(B)$ given by

$$
A \star B=\left(A \cap A^{*}\right) \otimes B+A^{0} \otimes C^{*}(B)
$$

One can verify that the inclusions $\phi_{F, G}$ defined above coincide with the natural inclusions

$$
T_{n_{w_{1}}} \star \cdots \star T_{n_{w_{k}}} \rightarrow T_{n_{w_{1}}} \star \cdots \star T_{n_{w_{i}}} \star T_{n_{w}} \star T_{n_{w_{1}+1}} \star \cdots \star T_{n_{w_{k}}}
$$

In fact the lexicographic product is an associative operation and the algebras $A(\Omega, \mu)$ can be viewed (unambiguously) as infinite lexicographic products of upper-triangular matrix algebras over the ordering $\Omega$. The following theorem is a corollary of Theorem 5 .

THEOREM 7. Let $G_{q}$ and $H_{q}$ for $q$ in $\mathbb{Q}$ be connected transitive antisymmetric digraphs with triangular digraph algebras $A\left(G_{q}\right)$ and $A\left(H_{q}\right)$. Then the lexicographic products

$$
\prod_{q \in \mathbb{Q}} \star A\left(G_{q}\right) \text { and } \prod_{q \in \mathbb{Q}} \star A\left(H_{q}\right)
$$

are isometrically isomorphic triangular operator algebras if and only if there is an order bijection $\pi$ such that the digraphs $G_{q}$ and $H_{\pi(q)}$ are isomorphic for all rationals $q$. 


\section{REFERENCES}

[1] W. B. Arveson. Analyticity in operator algebras. Amer. J. Math. 89 (1967), 578-642.

[2] A. P. Donsig. Semisimple triangular AF algebras. J. Functional Anal. 111 (1993), 323-349.

[3] A. P. Donsig and A. Hopenwasser. Order preservation in limit algebras. J. Functional Anal. 133 (1995), 342-394.

[4] A. Hopenwasser and S. C. Power. Classification of limits of triangular matrix algebras. Proc. Edinburgh Math. Soc. 36 (1992), 107-121.

[5] P. S. Muhly and B. Solel. Subalgebras of groupoid C*-algebras. J. für die Reine und Ange. Math. 402 (1989), 41-75.

[6] Y. T. Poon. A complete isomorphism invariant for a class of triangular UHF algebras. J. Operator Th. 27 (1992), 221-230.

[7] S. C. Power. Limit algebras: an introduction to subalgebras of $\mathrm{C}^{*}$-algebras. Pitman Research Notes in Mathematics vol 278. Longman Scientific and Technical, London-New York, 1992.

[8] S. C. Power. On the outer automorphism groups of triangular alternation limit algebras. J. Functional Anal. 113 (1993), 462-471.

[9] S. C. Power. Infinite lexicographic products of triangular algebras. Bull. London Math. Soc. 27 (1995), 273-277.

[10] J. Renault. A groupoid approach to $\mathrm{C}^{*}$-algebras. Lecture Notes in Math. 793. Springer, BerlinHeidelberg-New York, 1980. 